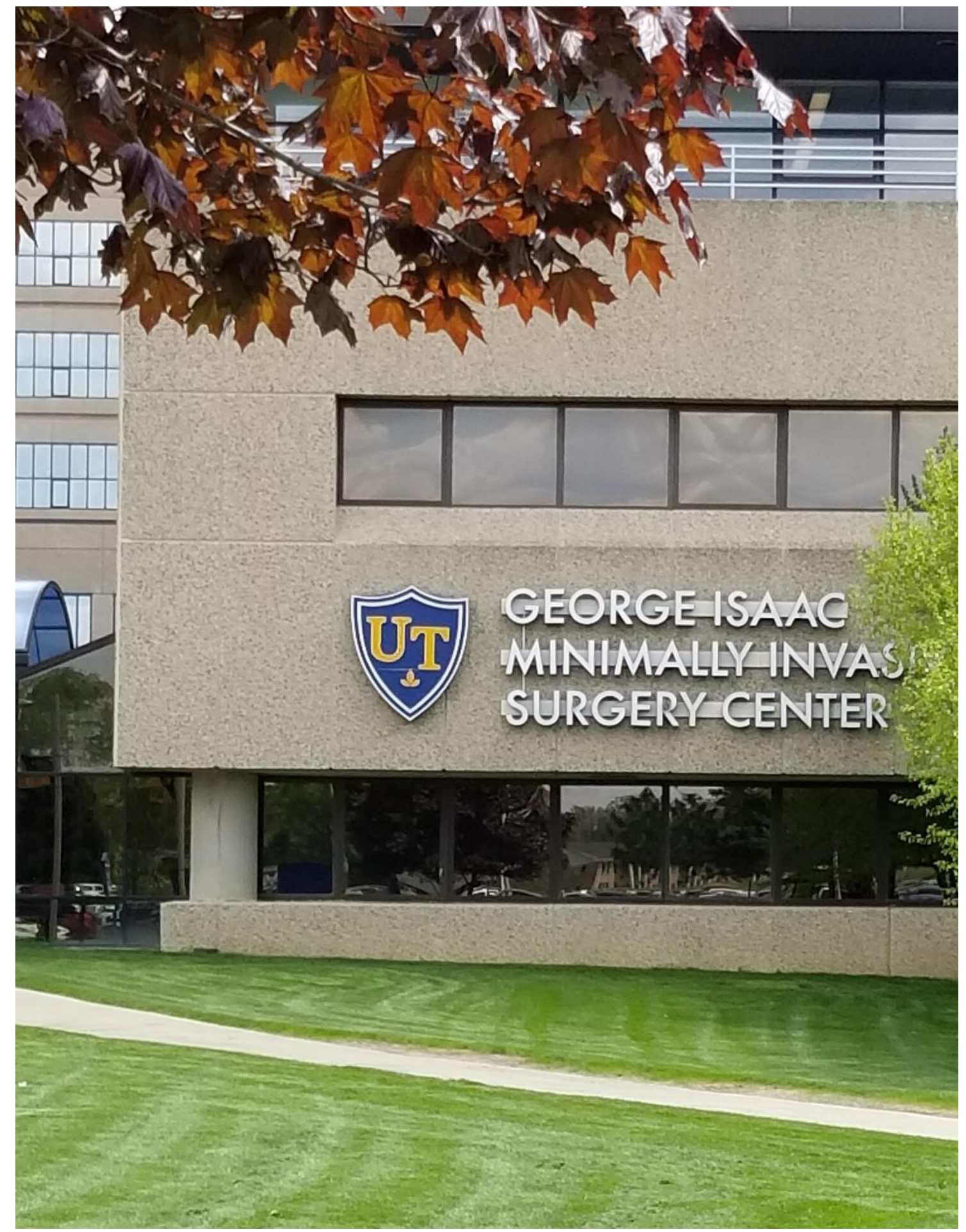




\title{
Streptococcus Intermedius Lung Abscess in a 17-year-old Male
}

\author{
Jason Levine ${ }^{a, 1}$ Karen Hovsepyan ${ }^{a}$ Daniel Lubarsky ${ }^{b}$ Deepa Mukundan ${ }^{c}$ and Jennifer Ruddy ${ }^{d}$
}

\begin{abstract}
${ }^{a}$ Department of Pediatrics, University of Toledo Health Science Campus, 3000 Arlington Ave, Toledo, OH 43614, USA, ${ }^{b}$ M.D. Candidate, Class of 2021, The University of Toledo Heath Science Campus, 3000 Arlington Ave., Toledo, OH 43614, USA., ${ }^{c}$ Department of Pediatric Infectious Disease, The University of Toledo Heath Science Campus, 3000 Arlington Ave., Toledo, OH 43614, USA, and ${ }^{d}$ Department of Pediatric Pulmonology, Cystic Fibrosis Center, ProMedica Toledo Children's Hospital, 2142 N Cove Blvd, Toledo, OH 43606, USA
\end{abstract}

\begin{abstract}
Streptococcus intermedius lung abscess and empyema is a chronic infectious process associated with a high morbidity and mortality. It is typically seen in adults, and is considered a rare disease process in the pediatric population. Our case describes a 17 year old male presenting with 3 months of cough associated with later development of chest and back pain. He was later found to have a severe empyema secondary to Streptococcus intermedius. This case underlines a rare diagnosis in the pediatric population, as well as an association with smoking and vaping.
\end{abstract}

\section{| marijuana | vaping-smoking | intermedius | empyema |}

A lthough it is not commonly seen in the world of pediatric medicine, Streptococcus intermedius, has the potential to cause high morbidity and mortality in all ages. Infection by Streptococcus intermedius is known to cause abscess formation in multiple areas of the body, including the brain, liver, and lungs. As in our case, the manifestation of this infection is quite insidious, indicating the need for a high index of suspicion, especially in the case of chronic cough. There is a significant amount of evidence being introduced that indicates smoking as a precipitant for common lung parenchymal infections, but there has only been minimal evidence specifically linking marijuana or vaping to Streptococcus intermedius empyema.

\section{Case report}

Patient Information: Age: 17 years old. Gender: Male. Ethnicity: Caucasian. Related Medical Problems: Chronic cough, shortness of breath, chest pain.

Objective. To discuss differential of chronic cough in an adolescent male, etiology and pathophysiology of Streptococcus intermedius empyema and the relationship between smoking, vaping, and empyema.

Case. 17-year-old Caucasian male with no significant past medical history presented with chronic cough for 3 months. Patient had multiple outpatient clinic visits with no improvement in his clinical status. He had progressively worsening symptoms and newly developed back pain which prompted him to visit a local emergency department. Chest imaging showed opacities and multifocal area of fluid collection in the right lung with associated mediastinal shift to the left (Figure 1A, 1B, and 1C). Patient was admitted to the pediatric intensive care unit for further management.

Family history was non-contributory. Past social history was negative for recent travel, animal exposure, sick contact, IV drug use, but was positive for marijuana smoking, vaping, and incarceration a few months prior to presentation.

Bloodwork showed the following: WBC $20.2 \times 10^{9} / \mathrm{L}, 89.4 \%$ segmented neutrophils, $4.8 \%$ lymphocytes, $5.8 \%$ monocytes; Na $131 \mathrm{mmol} / \mathrm{L}, \mathrm{K} 3.5 \mathrm{mmol} / \mathrm{L}, \mathrm{Cl} 99 \mathrm{mmol} / \mathrm{L}, \mathrm{CO}_{2} 22 \mathrm{mmol} / \mathrm{L}$, BUN $14 \mathrm{mg} / \mathrm{dL}$, Cr $0.67 \mathrm{mg} / \mathrm{dL}$, Glucose $120 \mathrm{mg} / \mathrm{dL}$, Ca $7.6 \mathrm{mg} / \mathrm{dL}$, Total protein $6.2 \mathrm{~g} / \mathrm{dL}$, Albumin $2.8 \mathrm{~g} / \mathrm{dL}$, Alkaline Phosphatase $91 \mathrm{U} / \mathrm{L}$, AST $35 \mathrm{U} / \mathrm{L}$, ALT $20 \mathrm{U} / \mathrm{L}$, Total bilirubin $0.4 \mathrm{mg} / \mathrm{dL}$; procalcitonin $2.13 \mathrm{ng} / \mathrm{mL}$, CRP $18.6 \mathrm{mg} / \mathrm{dL}$; Blood culture negative.

Once admitted to the PICU, patient was started on empiric Vancomycin and Ceftriaxone. Two chest tubes were placed in the two largest areas of fluid collection on day 1. Pleural fluid analysis of both samples showed total protein of $5.4 \mathrm{~g} / \mathrm{dL}$ and $5.3 \mathrm{~g} / \mathrm{dL}$, glucose $<10 \mathrm{mg} / \mathrm{dL}$ for both, and LDH of $4620 \mathrm{U} / \mathrm{L}$ and $3626 \mathrm{U} / \mathrm{L}$. Fluid culture isolated pan-sensitive Streptococcus intermedius. Transthoracic echocardiogram, duplex ultrasound of the internal jugular vein, MRA of carotid artery, were negative which effectively ruled out any thrombosis of neck vasculature or endocarditis as a source of infection. Vancomycin was discontinued, and patient was continued on monotherapy with ceftriaxone. Total of 5 doses of tissue plasminogen activator 4 milligrams each were administered through the chest tubes to lyse fibrin. Serial chest $\mathrm{x}$-rays were performed which showed progressive resolution of empyema (Figure 2A).

Chest tubes were removed on day 7. Patient was discharged home to complete total 3 weeks course of appropriate antibacterial therapy. Chest CT was performed 3 months after discharge showing complete resolution (Figures $2 \mathrm{~B}$ and $2 \mathrm{C}$ ).

Of note, later studies which were unremarkable include Total complement, serum immunoglobulins (IgM, IgG, and IgA), M. Tuberculosis sputum PCR and HIV antibody and antigen.

\section{Discussion}

Streptococcus intermedius is a gram positive, facultative aerobic, non-hemolytic coccus, which along with two other species Streptococcus constellatus and anginosus forms Streptococcus anginosus (Streptococcus milleri) group $(1,2)$. Streptococcus inter-

All authors contributed to this paper. ${ }^{1}$ To whom correspondence should be sent: Jason.Levine3@UToledo.Edu

The authors declare no conflict of interest. Submitted: 08/08/2019, published: 10/03/2019.

Freely available online through the UTJMS open access option 
medius is isolated most frequently from dental plaques, as it is part of our normal oral, respiratory tract and gastrointestinal microflora. It forms both rough and smooth colonies on Columbia agar; has Sialidase, Fucosidase, Galactosidase enzymes, and uniquely ferments both Raffinose and Mannitol (3). Additionally, it secretes pore forming cytolysins, which are believed to play a role in abscess formation and tissue destruction $(4,5)$. Despite being categorized as normal microflora of the oral cavity, Streptococcus intermedius is the most pathogenic agent of the Streptococcus anginosus group. The reason for its high pathogenicity is its ability to produce multiple types of abscesses as a solitary agent, commonly in brain and liver $(3,6,7)$. A majority of these are deep organ abscesses which may be more difficult to isolate due to accessibility (8).
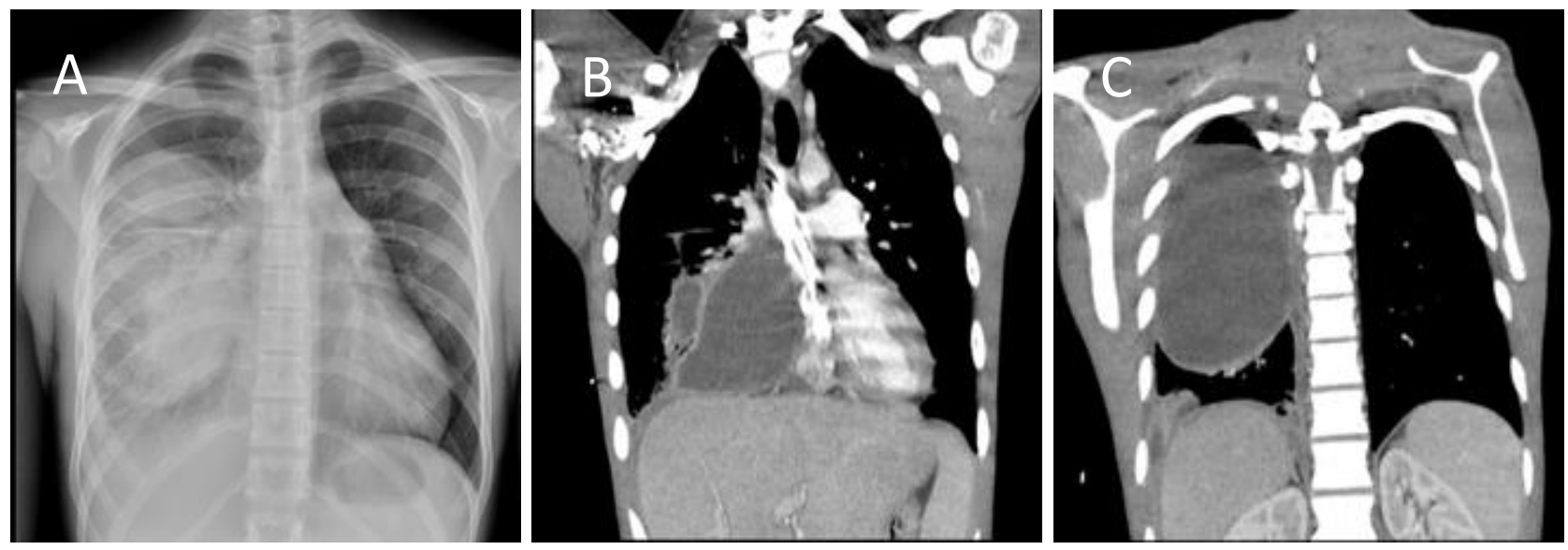

Figure 1. A: Right lung with significant density, without pneumothorax, B: loculated fluid collection anteriorly in right lung, left mediastinal shift, C: loculated fluid collection noted posteriorly in right lung.
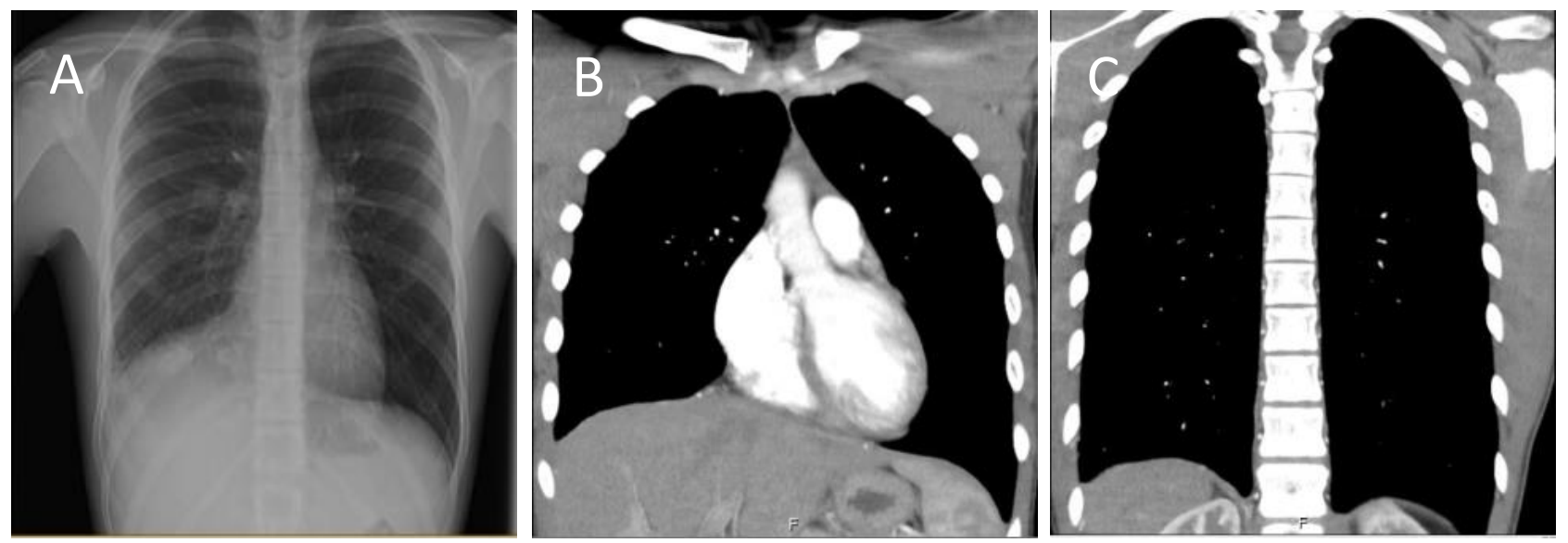

Figure 2. A: Progressive resolution of fluid collection, B: complete resolution of fluid collection 3 months after discharge, C: complete resolution of posterior fluid collection 3 months after discharge. . 
The majority of Streptococcus intermedius induced abscesses were reported in the adult population, who tend to have pulmonary infection and pleural effusion. The age became a reason for the hypothesis that aspiration of oral secretion may be a significant portion of the pathogenesis of S. intermedius infection (11). The most common type of Streptococcus intermedius infection in the pediatric population is a sinusitis (12), and it was presented that hematogenous dissemination is the main mechanism in children. This proposition was supported by the fact that in one third of cases with Streptococcus intermedius abscesses, blood culture was found to be positive $(7,12)$.

In spite of the progress made in healthcare today, morbidity and mortality from this infection remains high (13). Aggravating factors for increased mortality are: immunosuppression, diabetes mellitus, malignancies (especially gastrointestinal tract cancers), antibiotic use within 90-days and elder age $(57$ years \pm 14$)(11,14)$.

There are multiple etiologies and risk factors of Streptococcus intermedius infection that one must consider, such as the following:

Aspiration as a mechanism of illness of our patient was excluded, considering that he is young and previously healthy without neurological disorders, oropharyngeal disorders or dysmotility. In addition, he denied alcohol use, and he had a negative urine screen.

Considering that Streptococcus intermedius is a part of the microflora of the mouth, detailed dental examination was performed. He had normal dentition with no oral cavity lesions or injuries. Series of negative blood cultures made hematogenous dissemination for our patient also unlikely.

Patient's history included that he was incarcerated for about one week for behavioral issues. With this in mind, Tuberculosis workup was initiated, as empyema secondary to TB may occur. However, negative Interferon Gamma Release Assay excluded Mycobacterial infection. Patient's past medical history was significant for his habit to smoke marijuana and "vape". Review of the literature found multiple cases of tobacco smoking associated pneumonia in adolescents. It increases the risk of nasopharyngeal infection and pneumonia (OR 2.5 with CI 95\%) (15).

1. Whiley RA, Beighton D, Winstanley TG, Fraser HY, \& Hardie JM (1992) Streptococcus intermedius, Streptococcus constellatus, and Streptococcus anginosus (the Streptococcus milleri group): association with different body sites and clinical infections. J Clin Microbiol 30(1):243-244.

2. Whiley RA \& Beighton D (1991) Emended descriptions and recognition of Streptococcus constellatus, Streptococcus intermedius, and Streptococcus anginosus as distinct species. Int $J$ Syst Bacteriol 41(1):1-5.

3. Whiley RA, Fraser H, Hardie JM, \& Beighton D (1990) Phenotypic differentiation of Streptococcus intermedius, Streptococcus constellatus, and Streptococcus anginosus strains within the "Streptococcus milleri group". $J$ Clin Microbiol 28(7):1497-1501.

4. Giddings KS, Zhao J, Sims PJ, \& Tweten RK (2004) Human CD59 is a receptor for the cholesterol-dependent cytolysin intermedilysin. Nat Struct Mol Biol 11(12):1173-1178.

5. Nagamune $\mathrm{H}$, et al. (2004) The human-specific action of intermedilysin, a homolog of streptolysin $\mathrm{O}$, is dictated by domain 4 of the protein. Immunol 48(9):677-692.

6. Bantar C, et al. (1996) Species belonging to the "Streptococcus milleri" group: antimicrobial susceptibility and comparative prevalence in significant clinical specimens. J Clin Microbiol 34(8):2020-2022.

7. Claridge JE, 3rd, Attorri S, Musher DM, Hebert J, \& Dunbar S (2001) Streptococcus intermedius, Streptococcus constellatus, and Streptococcus anginosus ("Streptococcus milleri group") are of different clinical importance and are not equally associated with abscess. Infect Dis 32(10):1511-1515.
There are multiple mechanisms leading to increase in susceptibility to infection secondary to tobacco smoking including, but not limited to, smoking induced physiological-structural changes, increased bacterial pathogenicity, immune dysregulation, and vasoactive dysregulation of organs by bioactive agents of smoke (15).

Our patient denied any cigarette smoking but had history of marijuana use and vaping. There are case studies that suggest an association of marijuana smoking, and "vaping" to multiple pleuropulmonary infections $(16,17)$. Additionally, marijuana use has been reported in the patient history in previous case reports of Streptococcus intermedius pulmonary infection and mediastinal infection, although there is not yet any established causation between cannabis use and abscess formation from Streptococcus intermedius infections $(18,19)$

Considering the details of our case, literature reviews, patient's past medical history and habits, clinical and laboratory findings, there may be an association between this particular infection and smoking marijuana and/or vaping which needs to be further researched. Additionally, with persistent smoking, micro-aspiration of saliva or components of the oropharynx may occur. One may infer that this may also lead to Streptococcus intermedius pneumonia with subsequent Empyema formation.

\section{Conclusion.}

In summation, our goal is to present Streptococcus intermedius lung infection as a rare disease process in the pediatric population. In our case, we have concluded that smoking marijuana and vaping may be associated with Streptococcus intermedius pulmonary abscess or empyema. Physicians should be aware of this association and consider this in the differential for pediatric patients presenting with an infectious process involving the respiratory tract as well as history of marijuana smoking.

\section{Conflict of interest.}

Authors declare no conflict of interest.

\section{Authors' contributions}

JAL, KH, and DL wrote the manuscript, DM and JR revised the manuscript. All authors have read and approved the final document.

8. Young KA, Allaker RP, Hardie JM, \& Whiley RA (1996) Interactions between Eikenella corrodens and 'Streptococcus milleri-group' organisms: possible mechanisms of pathogenicity in mixed infections. Antonie Van Leeuwenhoek 69(4):371-373.

9. Jerng JS, et al. (1997) Empyema thoracis and lung abscess caused by viridans streptococci. Am J Respir Crit Care Med 156(5):1508-1514.

10. Wargo KA, McConnell VJ, \& Higginbotham SA (2006) A case of Streptococcus intermedius empyema. Ann Pharmacother 40(6):1208-1210.

11. Noguchi $S$, et al. (2015) The clinical features of respiratory infections caused by the Streptococcus anginosus group. Pulm Med 15:133.

12. Faden HS (2016) Infections Associated with Streptococcus intermedius in Children. Pediatr Infect Dis J 35(9):1047-1048.

13. Belko J, Goldmann DA, Macone A, \& Zaidi AK (2002) Clinically significant infections with organisms of the Streptococcus milleri group. Infect Dis $J$ 21(8):715723.

14. Wenzler $E$, et al. (2015) Clinical and microbiological outcomes in patients with Streptococcus anginosus group bacteraemia identified through use of a rapid microarray assay. J Med Microbiol 64(11):1369-1374.

15. Bagaitkar J, Demuth DR, \& Scott DA (2008) Tobacco use increases susceptibility to bacterial infection. Induc Dis 4:12.

16. Kumar AN, et al. (2018) Marijuana "bong" pseudomonas lung infection: a detrimental recreational experience. Case Rep 6(2):e00293.

17. Sussan TE, et al. (2015) Exposure to electronic cigarettes impairs pulmonary antibacterial and anti-viral defenses in a mouse model. PLoS One 10(2):e0116861. 
18. Catalya S, Komal B, Tulpule S, Raoof N, \& Sen S (2017) Isolated Streptococcus

intermedius pulmonary nodules. IDCases $8: 48-49$.

19. Hameed S, et al. (2017) Conglomerate mediastinal mass of a different etiology. 